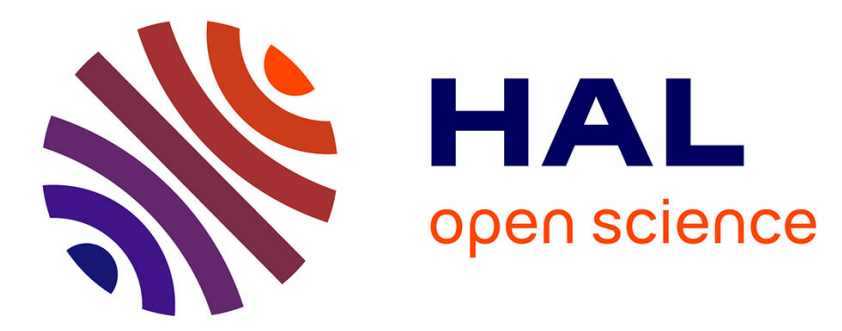

\title{
Impact of buffer layer and Pt thickness on the interface structure and magnetic properties in $(\mathrm{Co} / \mathrm{Pt})$ multilayers
}

\author{
M Bersweiler, Karine Dumesnil, D Lacour, Michel Hehn
}

\section{- To cite this version:}

M Bersweiler, Karine Dumesnil, D Lacour, Michel Hehn. Impact of buffer layer and Pt thickness on the interface structure and magnetic properties in $(\mathrm{Co} / \mathrm{Pt})$ multilayers. Journal of Physics: Condensed Matter, 2016, 28 (33), pp.336005. 10.1088/0953-8984/28/33/336005 . hal-02889081

\section{HAL Id: hal-02889081 https://hal.science/hal-02889081}

Submitted on 11 Dec 2020

HAL is a multi-disciplinary open access archive for the deposit and dissemination of scientific research documents, whether they are published or not. The documents may come from teaching and research institutions in France or abroad, or from public or private research centers.
L'archive ouverte pluridisciplinaire HAL, est destinée au dépôt et à la diffusion de documents scientifiques de niveau recherche, publiés ou non, émanant des établissements d'enseignement et de recherche français ou étrangers, des laboratoires publics ou privés. 
PAPER

\section{Impact of buffer layer and Pt thickness on the interface structure and magnetic properties in $(\mathrm{Co} / \mathrm{Pt})$ multilayers}

To cite this article: M Bersweiler et al 2016 J. Phys.: Condens. Matter 28336005

View the article online for updates and enhancements.
Related content

\begin{tabular}{l} 
- Enhancement of perpendicular magnetic \\
anisotropy and coercivity in ultrathin \\
$\underline{\text { Ru/Co/Ru films through the buffer layer }}$ \\
engineering \\
Alexander G Kolesnikov, Maxim E Stebliy, \\
Alexey V Ognev et al. \\
- Perpendicular magnetic anisotropy and \\
\hline microstructure properties of nanoscale \\
\hline Co/Au multilayers \\
C Rizal and E E Fullerton \\
- Magnetic anisotropy in metallic multilavers \\
M T Johnson, P J H Bloemen, F J A den \\
Broeder et al.
\end{tabular}

\section{Recent citations}
Heterolytic dissociative adsorption state of dihydrogen favored by interfacial defects Zhenjun Song et al




\title{
Impact of buffer layer and Pt thickness on the interface structure and magnetic properties in $(\mathrm{Co} / \mathrm{Pt})$ multilayers
}

\author{
M Bersweiler, K Dumesnil, D Lacour and M Hehn \\ Institut Jean Lamour (UMR CNRS 7198), Université de Lorraine, B.P. 70239, \\ Vandoeuvre-lès-Nancy F-54506, France \\ E-mail: michel.hehn@univ-lorraine.fr
}

Received 22 February 2016, revised 29 April 2016

Accepted for publication 31 May 2016

Published 28 June 2016

\begin{abstract}
The influence of Pt thickness on the interface structure (roughness/intermixing) and magnetic properties has been investigated for $(\mathrm{Co} / \mathrm{Pt})$ multilayers sputtered on a $\mathrm{Pt}$ or a thin oxide $\left(\mathrm{MgO}\right.$ or $\left.\mathrm{AlO}_{x}\right)$ buffer layer. When Pt thickness increases from $1.2 \mathrm{~nm}-2.2 \mathrm{~nm}$, we observe that the effective anisotropy increases with the Pt thickness, simultaneously with the decrease of roughness, i.e. the occurrence of sharper interfaces. Perpendicular magnetic anisotropy (PMA) is still achieved on the oxide buffer layers, but with a lower effective anisotropy correlated to more perturbed interfaces. The detailed analysis of the saturation magnetization shows that: (i) $M_{\mathrm{S}}$ is significantly enhanced in the case of rough/intermixed interfaces, which is attributed to and discussed in the framework of Pt induced polarization, (ii) the change in volume dipolar anisotropy is the main factor responsible for the reduction of $K_{\text {eff }}$ for systems grown on oxides. Beyond the major role of volume dipolar contribution that reduces PMA, a supplemental positive contribution promoting PMA can be invoked for rough interfaces and large $M_{\mathrm{S}}$ (deposit on oxide). This contribution is consistent with a dipolar surface anisotropy term and increases for rough interfaces, in contrast to the Néel surface anisotropy. These opposite variations may interestingly lead to an enhanced anisotropy in $(\mathrm{Co} / \mathrm{Pt})$ stackings grown on oxides compared to systems deposited on Pt, i.e. with sharper interfaces.
\end{abstract}

Keywords: perpendicular magnetic anisotropy, structural properties, magnetic properties, $\mathrm{Co} / \mathrm{Pt}$ multilayers

(Some figures may appear in colour only in the online journal)

\section{Introduction}

Magnetic materials/systems displaying perpendicular magnetic anisotropy (PMA) exhibit several key advantages over those with in-plane magnetization for the media storage industry, especially for spintronics and memory applications: higher thermal stability and smaller domain wall widths [1, 2], lower threshold current for spin-transfer switching [1] and for current-induced domain wall motion [3].

The common phenomenological approach to the effective anisotropy $K_{\text {eff }}$ in thin films or multilayers is to consider both volume $K_{\mathrm{v}}$ and surface $K_{\mathrm{s}}$ contributions: $K_{\text {eff }} t=K_{\mathrm{v}} t+2 K_{\mathrm{s}}$, where $t$ is the thickness of the magnetic material. Volume contributions correspond to magneto-crystalline $(m c)$, magneto-elastic $(m e)$ and shape anisotropies $\left(-2 \pi M_{\mathrm{s}}^{2}\right)$, while surface/interface contributions are generally attributed to broken symmetry [4] and/or surface magneto-elastic strains [5, 6]. The shape anisotropy always favors in-plane magnetization and is often the dominant volume contribution. Achieving a perpendicular magnetic anisotropy is thus possible when (i) positive volume $m c$ and $m e$ contributions, or when (ii) positive surface/interface contributions can overcome the shape anisotropy. The first case (i) is encountered for chemically ordered $\mathrm{L}_{0}$ alloy (FePt, FePd, CoPt) films that exhibit suitable orientation [7] and for materials in which growth-induced strains can lead to a large enough positive magnetoelastic 
contribution [8, 9]. However the large majority of systems exhibiting PMA are magnetic multilayers in which surface/ interface effects are at the origin of PMA. Among these, Co-based multilayers (Co/Au [10], $\mathrm{Co} / \mathrm{Cu}$ [11], Co/Pt [12], $\mathrm{Co} / \mathrm{Pd}$ [13], $\mathrm{Co} / \mathrm{Ni}$ [14]) have attracted considerable interest now for more than two decades, one of the main objectives being to unravel and identify the mechanisms promoting the PMA at surfaces/interfaces.

In the $\mathrm{Co} / \mathrm{Pt}$ system, most studies now agree on the essential role of the so-called Néel anisotropy, i.e. the PMA contribution associated with the enhanced anisotropy of the orbital moment due symmetry breaking at the interfaces [15]. This contribution is strongly dependent on the crystal orientation, the $\langle 111\rangle$ texture giving rise to the highest anisotropy constants [16], and to the morphology of the interfaces. This latter point has been explored by analyzing the role of various key parameters expected to modify the interface structure (deposition methods [16], nature and pressure of the sputtering gas $[13,17,18]$, annealing temperature [12], etc) on the effective anisotropy. However, the precise role of roughness/intermixing remains relatively unclear since enhanced PMA has been reported for chemically mixed interfaces, likely associated with the formation of a chemically ordered CoPt alloy $[12,18]$, while other studies conclude that there is a clear reduction of PMA when leaving the ideal sharp interfaces [19]. The theoretical formalism developed by Bruno et al $[6,20$, $21]$ contributes to the debated role of roughness/intermixing. In treating the interface roughness as a succession of sharp steps and flat areas, the authors show that roughness may both (i) reduce PMA in reducing the asymmetric character of the interfaces and thus the Néel surface anisotropy and (ii) favor PMA in giving rise to a positive dipolar surface anisotropy contribution.

A precise determination of film roughness/intermixing using X-ray and electron diffraction is thus a necessary step in correlating microstructural disorder with magnetic properties, as claimed by several authors $[22,23]$. But, up to now, a few exceptions apart [23, 24], most of the studies have relied on relatively indirect characterization of interfaces, as for example the measurement of electrical resistivity or an induced Pt moment [12, 19].

In this paper, the interface structure in $(\mathrm{Co} / \mathrm{Pt})$ multilayers with thin Co layers (approximately 3ML thick) and only four bilayer repeats has been directly and carefully analyzed via $\mathrm{x}$-ray reflectivity and correlated with magnetic properties, especially PMA and saturation magnetization. The main objectives here were (i) to focus on the role of the Pt thickness $(1.2 \mathrm{~nm}-2.2 \mathrm{~nm}$ range), a parameter that has been rarely considered $[16,25,26]$ or mostly for the investigation of interlayer coupling [27, 28], and (ii) to compare the deposition on an usual metallic buffer with the deposition on an intermediate thin oxide layer. As pointed out in a few recent studies [24, 29], this latter case is of interest for the development of perpendicularly magnetized top electrodes in magnetic tunnel junctions (MTJs) and for other spintronic devices: spin injection in semiconductors or devices where a metallic current-shunting path should be avoided. The first section of the paper details the experimental techniques used for the study. The second part describes the experimental results, i.e. structural and magnetic analyses, that are discussed to highlight the correlation between roughness/intermixing and magnetic properties in these systems. The variations of effective anisotropy are presented in a first step. The analysis of saturation magnetization then permits us to explore the role of the interface structure on induced $\mathrm{Pt}$ moments and to extract the volume dipolar contribution from anisotropy. We thus show that if volume dipolar terms largely contribute to the effective anisotropy, supplemental contributions associated with the rough/intermixed interface, and consistent with surface dipolar terms, may also enhance the anisotropy.

\section{Experimental details}

Several sets of $(\mathrm{Co} / \mathrm{Pt})$ multilayers with various buffer and capping layers (table 1) have been synthetized and investigated. The $(\mathrm{Co} / \mathrm{Pt})$ stackings have been sputtered on a silicon substrate covered by a $100 \mathrm{~nm}$-thick amorphous thermal silicon oxide. After a soft etching of the substrate under pure Ar plasma, a $5 \mathrm{~nm}$-thick tantalum layer has been first deposited in order to favor the good adhesion of Pt and promote its (1 111 ) texture with a deposition rate of $1.2 \AA^{-1}$. Then the multilayer is deposited with a typical flux deposition of $0.6 \AA \mathrm{s}^{-1}$ for $\mathrm{Pt}$ and $0.09 \AA \mathrm{s}^{-1}$ for Co. The details of the different series are given below:

Set A:

Substrate $/ \mathrm{Ta}(5) / \operatorname{Pt}(5) /\left(\operatorname{Co}\left(t_{\mathrm{Co}}\right) / \operatorname{Pt}(1.8)\right] 4 / \operatorname{Pt}(5)$

Sets B and C:

Substrate $/ \mathrm{Ta}(5) / \mathrm{Pt}(5) /\left[\mathrm{Co}(0.58) / \mathrm{Pt}\left(t_{\mathrm{Pt}}\right)\right]_{3} / \operatorname{Co}(0.58) / \mathbf{X}(\mathbf{2 . 5})$

Sets D and E:

Substrate/ $\mathrm{Ta}(5) / \mathrm{Pt}(5) / \mathbf{X}(\mathbf{2 . 5}) /\left[\mathrm{Co}(0.58) / \mathrm{Pt}\left(t_{\mathrm{Pt}}\right)\right]_{4} / \mathrm{Pt}(5)$

Numbers in parentheses give the nominal thicknesses in nanometers and $\mathrm{X}$ corresponds to $\mathrm{MgO}$ (sets $\mathrm{B}$ and $\mathrm{D}$ ) or $\mathrm{AlO}_{x}$ (sets $\mathrm{C}$ and $\mathrm{E}$ ), the thickness of which stands in the usual $(2-3 \mathrm{~nm})$ range for the insulating barrier in MTJs. Such stackings are thus similar to the bottom (sets B and C) or top (sets D and E) electrodes that may constitute MTJs with $\mathrm{MgO}$ or $\mathrm{AlO}_{x}$ barriers. The Pt thickness has been varied between $1.2 \mathrm{~nm}$ and $2.2 \mathrm{~nm}$. Samples from set A have been synthetized prior to other samples to investigate the magnetic anisotropy as a function of the Co thickness (in the $0.29 \mathrm{~nm}-2.23 \mathrm{~nm}$ range) for $1.8 \mathrm{~nm}$-thick Pt layers, and thus to determine the optimal Co thickness for sets B-E.

The structural analysis of samples from sets B-E has been performed by X-ray reflectivity using a PanAnalytical X'Pert Pro diffractometer and working with the $K_{\alpha 1}$ transition of copper $(\lambda=1.54056 \AA)$. The fit of reflectivity data has been made via the commercial X'pert Reflectivity software based on the Parratt formalism [30]. Large angle X-ray reflectivity measurements have also been performed for similar $(\mathrm{Co} / \mathrm{Pt})$ stackings, but with more bilayer repeats and without any thick 
Table 1. Description of the various sets of $(\mathrm{Co} / \mathrm{Pt})$ multilayers investigated in this study.

\begin{tabular}{llll}
\hline & Buffer & $(\mathrm{Co} / \mathrm{Pt})$ stacking & Capping \\
\hline Set A & $\mathrm{Ta}(5) / \operatorname{Pt}(5)$ & $\left(\mathrm{Co}\left(t_{\mathrm{Co}}\right) / \mathrm{Pt}(1.8)\right)_{4}$ & $\operatorname{Pt}(5)$ \\
Set B & $\mathrm{Ta}(5) / \mathrm{Pt}(5)$ & $\left(\mathrm{Co}(0.58) / \mathrm{Pt}\left(t_{\mathrm{Pt}}\right)\right)_{3} / \mathrm{Co}$ & $\mathrm{MgO}(2.5)$ \\
Set C & $\mathrm{Ta}(5) / \mathrm{Pt}(5)$ & $\left(\mathrm{Co}(0.58) / \mathrm{Pt}\left(t_{\mathrm{Pt}}\right)\right)_{3} / \mathrm{Co}$ & $\mathrm{AlO} x(2.5)$ \\
Set D & $\mathrm{Ta}(5) / \mathrm{Pt}(5) / \mathrm{MgO}(2.5)$ & $\left(\mathrm{Co}(0.58) / \mathrm{Pt}\left(t_{\mathrm{Pt}}\right)\right)_{4}$ & $\operatorname{Pt}(5)$ \\
Set E & $\mathrm{Ta}(5) / \mathrm{Pt}(5) / \mathrm{AlO}_{x}(2.5)$ & $\left(\mathrm{Co}(0.58) / \mathrm{Pt}\left(t_{\mathrm{Pt}}\right)\right)_{4}$ & $\operatorname{Pt}(5)$ \\
\hline
\end{tabular}

Note: Numbers in parentheses give the nominal thicknesses in nanometers.

Pt buffer or cap layers; they confirmed the expected (111) texture in these growth conditions.

The magnetic properties of $(\mathrm{Co} / \mathrm{Pt})$ multilayers have been investigated at room temperature using a vibrating sample magnetometer with an available field range of $\pm 25 \mathrm{kOe}$. Magnetization versus field $(M(H))$ loops have been recorded for magnetic fields applied both perpendicular and parallel to the sample surface. The corresponding effective anisotropy constants have been determined using the 'area method' [31] which consists in determining the area difference between the in-plane and perpendicular hysteresis curves in one $M(H)$ quadrant.

\section{Results and discussion}

\subsection{Structural properties}

Figure 1 presents typical examples of $\mathrm{x}$-ray reflectivity data obtained within each $(\mathrm{Co} / \mathrm{Pt})$ set (sets $\mathrm{B}-\mathrm{E})$ for a nominal $\mathrm{Pt}$ thickness $t_{\mathrm{Pt}}=1.13 \mathrm{~nm}$. Despite the rather high complexity of these layered systems, an excellent agreement could be obtained between the experimental reflectivity curves and the results of the fitting process. Information on the layers thicknesses and the interfaces structure can thus be extracted with a relatively high degree of confidence. One has, however, to underline here that $\mathrm{x}$-ray reflectometry in specular geometry is sensitive to the electronic density profile along the normal to the sample surface. The method is thus highly sensitive to the quality of interfaces but does not permit us to distinguish between roughness and intermixing effects at the interfaces. The 'roughness' used in the following is thus a generic term to characterize the interface quality/sharpness. Other complementary methods, such as transmission electron microscopy and nuclear magnetic resonance, would be necessary to go further into the interface analysis.

The thickness values extracted from the fitting process show that the Co thickness exhibits very small dispersion in a given set of samples with an average value of $0.58 \mathrm{~nm}$ for set $\mathrm{D}$ and $0.61 \mathrm{~nm}$ for sets $\mathrm{B}, \mathrm{C}$ and $\mathrm{E}$. The Pt thicknesses exhibit a maximum dispersion of only $6 \%$ between the various sets. The fitted values for Co and Pt thicknesses are used for the various analyses presented below.

Co and Pt roughnesses obtained from the reflectivity data (top roughness of each layer) are reported versus the Pt thickness in figures 2(a) and (b) respectively. For set E and for the
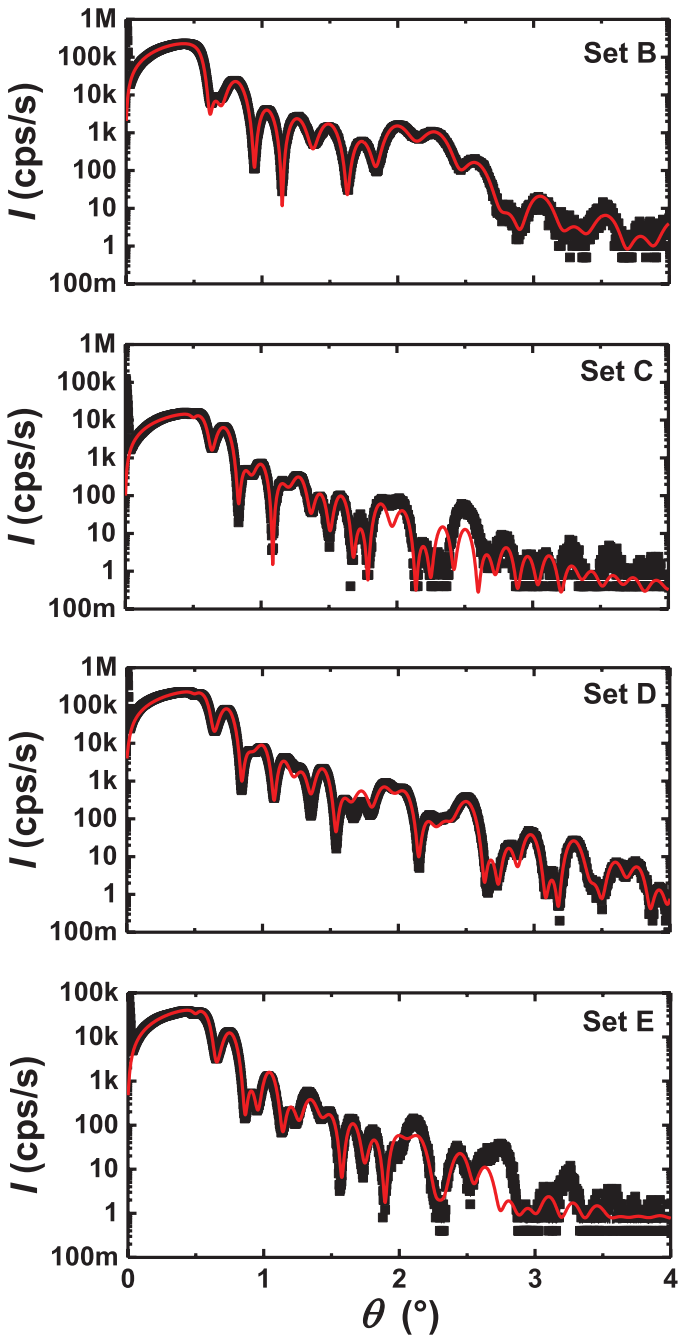

Figure 1. X-ray reflectivity data (black curves) and fit of the experimental data using X'Pert Reflectivity (red curves) for samples with nominal thicknesses $t_{\mathrm{Co}}=0.58 \mathrm{~nm}$ and $t_{\mathrm{Pt}}=1.13 \mathrm{~nm}$ in the sets B-E.

lowest Pt thickness, it has not been possible to extract a value for the Co roughness with reasonable error bars because of the poorer quality of the fits obtained for samples grown on $\mathrm{AlO}_{x}$ compared to the other sets of samples. For the Pt layers (figure 2(b)), no variation versus the Pt thickness could be reasonably extracted. However, for the Co layers (figure 2(a)), the general trend is a clear decrease of the roughness when the Pt thickness increases. This tendency is observed for each set despite some scattering of the results, likely related to the complexity of the fitting process.

Concerning the role of the buffer layer, $(\mathrm{Co} / \mathrm{Pt})$ multilayers sputtered on a Pt buffer (sets B and C) exhibit significantly smaller roughnesses than those sputtered on $\mathrm{MgO}$ (set D) and $\mathrm{AlO}_{x}$ (set E), this latter appearing to particularly damage the quality of the interfaces. The Co roughness is increased by nearly $50 \%$ in the case of $\mathrm{MgO}$ and $\mathrm{AlO}_{x}$ buffers. Given the small thickness of the Co layers, such roughness values mean that the $\mathrm{Co} / \mathrm{Pt}$ interfaces are then poorly defined with a high degree of roughness and/or inter-mixing. The situation 

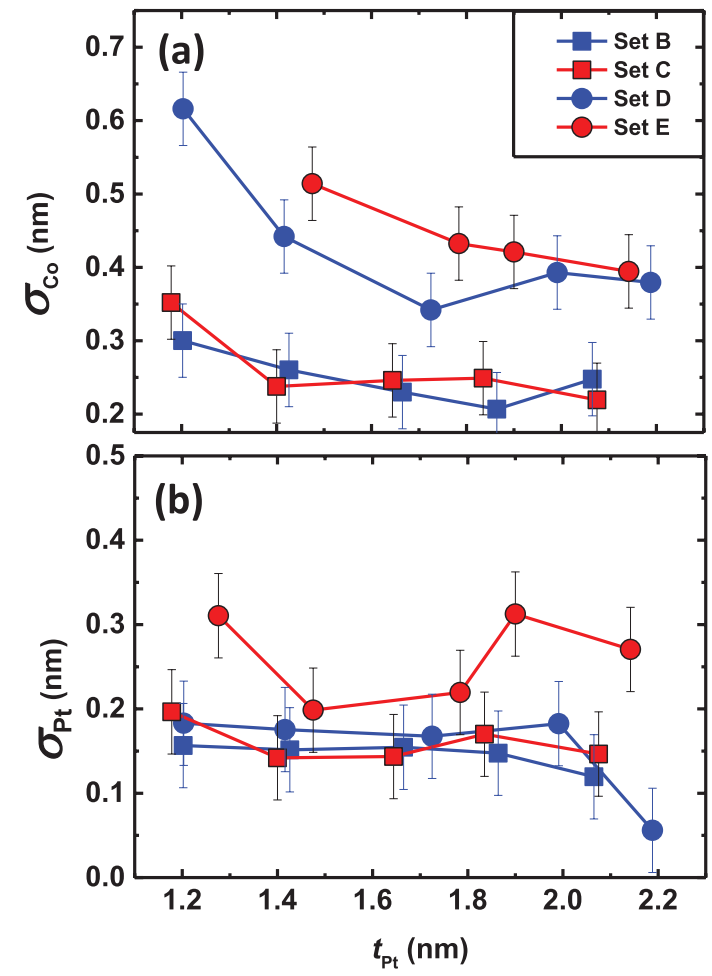

Figure 2. Variation of the $\mathrm{Co}\left(\sigma_{\mathrm{Co}}\right)$ and $\mathrm{Pt}\left(\sigma_{\mathrm{Pt}}\right)$ roughnesses (respectively in (a) and (b)) extracted from the fit of $\mathrm{x}$-ray reflectivity data versus the Pt thickness for the sets B-E.

is better for Pt layers, the larger thickness of which probably helps in smoothing the interface, but a large roughness is still obtained on the $\mathrm{AlO}_{x}$ buffer. This difference between metallic and oxide buffers is attributed to the difference between the surface energies of the various buffer layers: a thin continuous metallic layer is more likely obtained when it is deposited on a metallic buffer layer with high surface energy $\left(\approx 2 \times 10^{-3} \mathrm{erg} \mathrm{cm}^{-2}\right)$ than on an insulating buffer layer with much smaller surface energy (generally close or smaller than $1 \times 10^{-3} \mathrm{erg} \mathrm{cm}^{-2}$ ) [32]. The room temperature deposition on insulators promotes the growth of islands, discontinuous layers leading to rough interfaces. In our specific case, the poorer interface quality observed for deposition on $\mathrm{AlO}_{x}$ is also consistent with the smaller surface energy generally reported for aluminum oxides $\left(0.6 \times 10^{-3} \mathrm{erg} \mathrm{cm}^{-2}\right) \mathrm{com}$ pared to $\mathrm{MgO}\left(1.16 \times 10^{-3} \mathrm{erg} \mathrm{cm}^{-2}\right)$ [33].

\subsection{Magnetic properties of (Co/Pt) stackings}

As a first step, the magnetic properties of set A samples (fixed Pt thickness) have been analyzed versus the Co thickness to extract the surface $\left(K_{\mathrm{S}}\right)$ and volume $\left(K_{\mathrm{V}}\right)$ contributions to the anisotropy constant, defined as $K_{\text {eff }} \times t_{\mathrm{Co}}=K_{\mathrm{V}} \times t_{\mathrm{Co}}+2 K_{\mathrm{S}}$ (figure 3). Values of $K_{\mathrm{S}}=0.72 \mathrm{erg} \mathrm{cm}^{-2}$ and $K_{\mathrm{V}}=-13 \times 10^{6}$

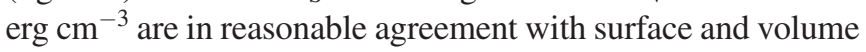
anisotropy constants reported for (111) textured $(\mathrm{Co} / \mathrm{Pt}) \mathrm{mul}-$ tilayers [31]. Two regimes are distinguished when varying the Co thickness: (i) in-plane magnetization for $t_{\mathrm{Co}}>1.1 \mathrm{~nm}$, where the demagnetizing field, the main contribution to the volume

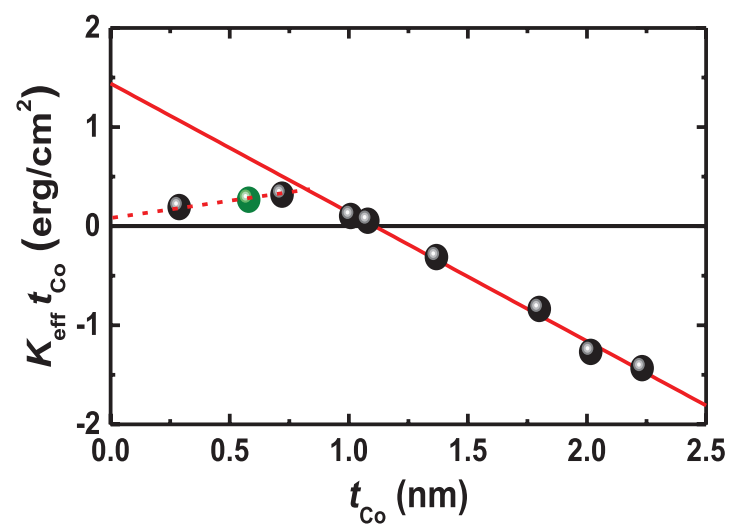

Figure 3. Room temperature effective anisotropy multiplied by the Co thickness versus the Co thickness for $(\mathrm{Co} / \mathrm{Pt})$ multilayers sputtered on a Pt buffer (set A). The green point corresponds to the value for $t_{\mathrm{Co}}=0.58 \mathrm{~nm}$, i.e. the nominal Co thickness of studied samples, assuming a linear dependence for thickness below $0.75 \mathrm{~nm}$ (dashed line). The solid line corresponds to the linear fit for thicknesses above $0.75 \mathrm{~nm}$.

anisotropy, dominates the interface effects; (ii) perpendicular magnetization for $t_{\mathrm{Co}}<1.1 \mathrm{~nm}$, where the interface effects are dominant. A reduction in the effective anisotropy (deviation from the linear behavior, the continuous red line) is observed at low Co thickness (less than $0.75 \mathrm{~nm}$ ), most likely due to the reduction of surface anisotropy associated with the degradation of interfaces when decreasing the Co thickness [16, 34, 35]. From this preliminary analysis, a fixed Co nominal thickness of $0.58 \mathrm{~nm}$ has been chosen, yielding PMA with an effective anisotropy constant of $4.6 \times 10^{6} \mathrm{erg} \mathrm{cm}^{-3}$ (green point in figure 3 , determined from the linear fit for thickness below $0.75 \mathrm{~nm}$ ). In the following, Pt thickness as well as buffer and capping layers have been changed.

Hysteresis loops measured at room temperature, for an applied field perpendicular $(\perp)$ and parallel (//) to the sample surface, are presented in figure 4 for the various $(\mathrm{Co} / \mathrm{Pt}) \mathrm{mul}-$ tilayers from sets B-E. The corresponding effective anisotropy constants are reported in figure 5.

The samples sputtered on the Pt buffer (sets B and C) exhibit perpendicular easy magnetization direction over the entire investigated $\mathrm{Pt}$ thickness range, with equal saturation magnetization values and fully remanent magnetization, despite a small in-plane component observed for the smallest Pt thickness. Their effective anisotropy constants are very close and both slightly decrease from 5 to $2 \times 10^{6} \mathrm{erg} \mathrm{cm}^{-3}$ when decreasing the Pt thickness. It can be noticed that the range of $K_{\text {eff }}$ is consistent with $K_{\text {eff }}$ values reported for previous works on $(\mathrm{Co} / \mathrm{Pt})$ multilayers $[36,37]$. This tendency for $K_{\text {eff }}$ to decrease with the Pt thickness is also consistent with results obtained in $(\mathrm{Co}(0.3 \mathrm{~nm}) / \mathrm{Pt}(\times \mathrm{nm}))$ multilayers $(x \in(0.2-2) \mathrm{nm})$ [16]. In this latter study $K_{\text {eff }}$ is, however, almost constant down to $1 \mathrm{~nm}$ and exhibits a larger value $\left(10 \times 10^{6} \mathrm{erg} \mathrm{cm}^{-3}\right)$, which could be related to the larger number of bilayer repeats.

The samples sputtered on oxide buffers (sets D and E) exhibit a transition from perpendicular to an in-plane easy magnetization direction when decreasing the Pt thickness. 
Set B: Pt/[Co/Pt $]_{3} / \mathrm{Co} / \mathrm{MgO}$
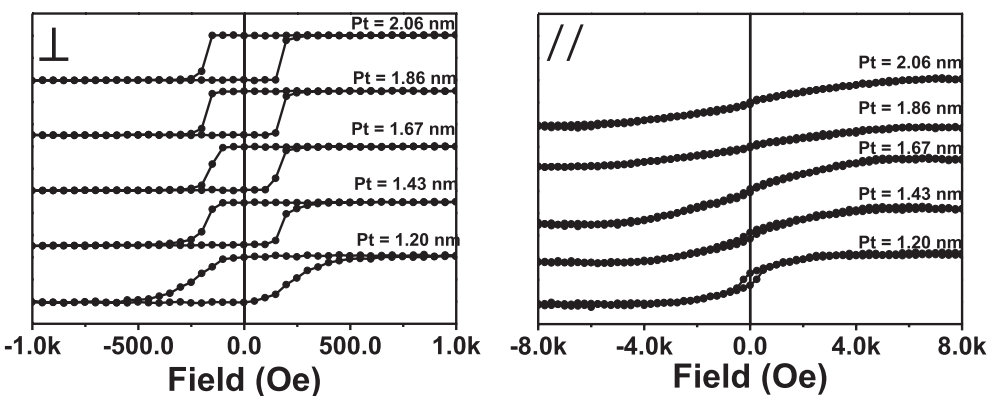

Set C: $\mathrm{Pt} /[\mathrm{Co} / \mathrm{Pt}]_{3} / \mathrm{Co} / \mathrm{AlO}_{x}$
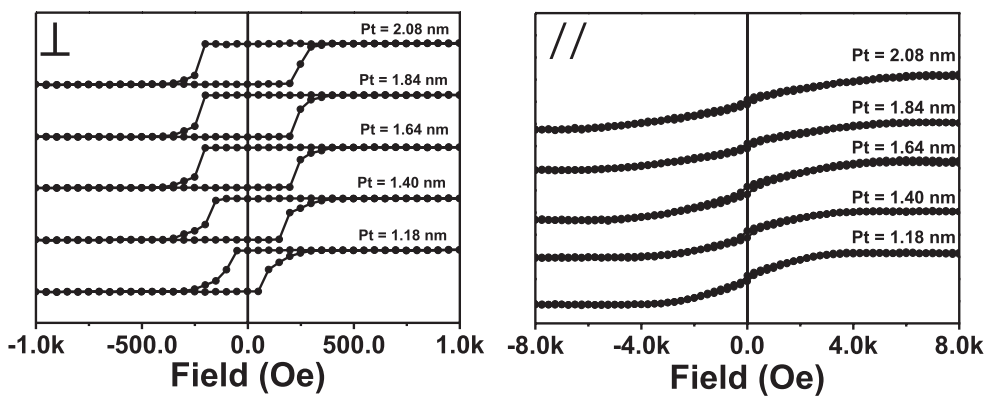

Set $\mathrm{D:} \mathrm{MgO} /[\mathrm{Co} / \mathrm{Pt}]_{4} / \mathrm{Pt}$
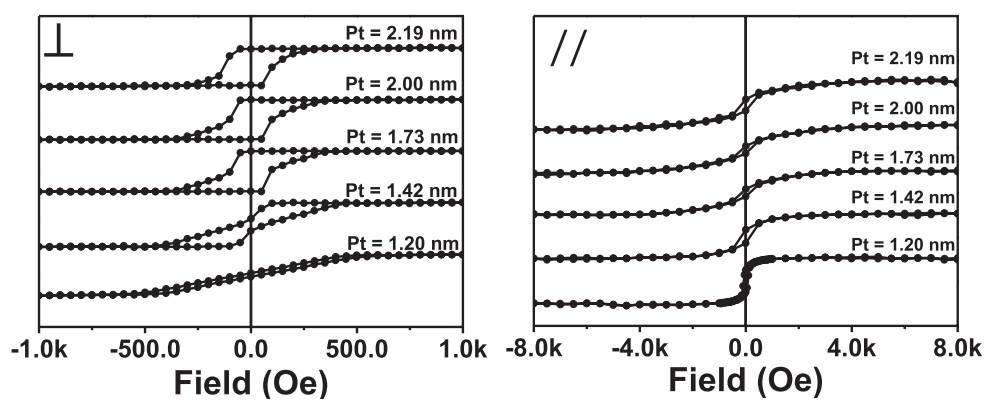

Set E: $\mathrm{AlO} \times x /[\mathrm{Co} / \mathrm{Pt}]_{4} / \mathrm{Pt}$
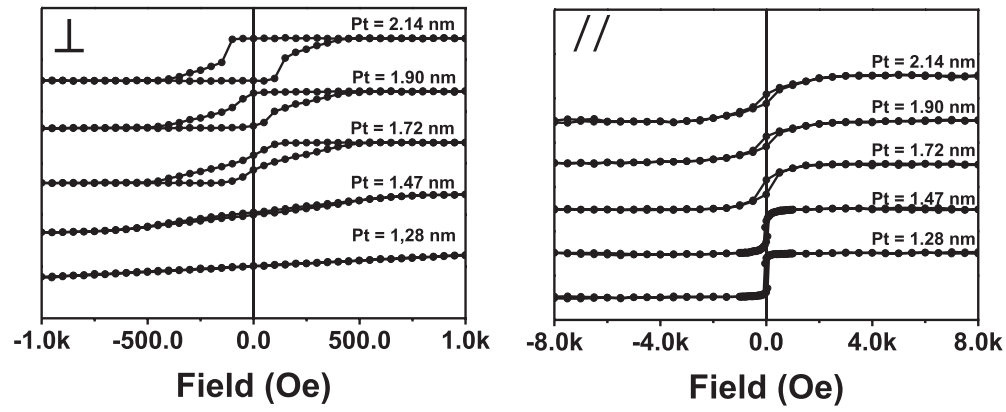

Figure 4. $M(H)$ loops for the out-of-plane (left) and in-plane (right) configurations measured at room temperature for $(\mathrm{Co} / \mathrm{Pt}) \mathrm{multilayers}$ sputtered on various buffers and with different capping layers ( $\mathrm{Pt}, \mathrm{MgO}$ or $\mathrm{AlO}_{x}$ ).

$K_{\text {eff }}$ decreases when decreasing the Pt thickness but it is significantly reduced compared to samples sputtered on Pt, and eventually becomes negative for the thinnest Pt layers. The reduction in effective anisotropy is obviously more important for samples sputtered on $\mathrm{AlO}_{x}$ (set E) for which the in-plane easy magnetization also appears for thicker Pt layers $(1.53 \mathrm{~nm})$.

The larger effective anisotropy for $(\mathrm{Co} / \mathrm{Pt})$ sputtered on $\mathrm{Pt}$ can be first explained by a likely better (111) texture which is known to promote the PMA [13, 38, 39]. This hypothesis is 


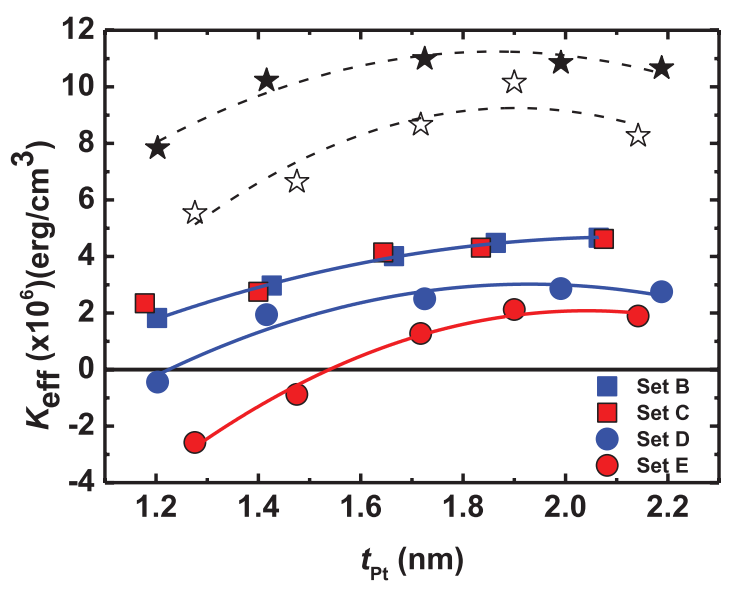

Figure 5. Variations of the effective anisotropy constants with the Pt thickness for the sets B-E. The star symbols correspond to effective anisotropy values for sets D (filled stars) and E (empty stars), calculated for volume dipolar contributions identical to sets $\mathrm{B}$ and $\mathrm{C}$. The solid and dashed lines are guides for the eyes.

strengthened by the larger coercive fields and the more abrupt magnetization reversal (figure 4) observed for the $(\mathrm{Co} / \mathrm{Pt}$ ) multilayers grown on Pt. The coercive fields measured for the samples grown on $\mathrm{Pt}$ are close to 200 Oe, i.e. similar to or slightly smaller than the values reported by other authors for these Co and Pt thicknesses [27-29, 34]. Some studies have reported on the non-monotonic/oscillatory variation of $H_{\mathrm{c}}$ as a function of $t_{\mathrm{Pt}}$, which has been attributed to the oscillatory interlayer coupling in $(\mathrm{Co} / \mathrm{Pt})$ multilayers $[27,28]$. No similar behavior could be extracted from the current study where the investigated Pt thickness range is rather limited.

But beyond the effect of texture, the variation of effective anisotropy constants versus Pt thickness, depending on the nature of the buffer layer, can be interestingly compared to the structural characteristics reported in the previous paragraph.

- The analysis of the Co roughness has shown: (i) a significant upward shift between sets B-C (grown on Pt) and sets $\mathrm{D}-\mathrm{E}$ (grown on $\mathrm{MgO}$ and $\mathrm{AlO}_{x}$ ), (ii) a clear tendency to decrease by increasing the Pt thickness for sets D-E, (iii) a slight tendency to decrease for sets B-C.

- The effective anisotropy exhibits opposite variations with (i) a significant shift downward between sets B-C and sets $\mathrm{D}-\mathrm{E}$, (ii) a clear increase by increasing the $\mathrm{Pt}$ thickness for all sets. It appears that a larger roughness obtained in decreasing the Pt thickness and/or in using an oxide buffer yield to a decrease in effective anisotropy.

This observed correlation between the roughness and the effective anisotropy is consistent with the usual expected role of roughness/interdiffusion: the increase in roughness/ interdiffusion yields a reduction of the symmetry breaking at the interfaces and consequently a reduction of the Néel surface anisotropy contribution to the PMA.

In order to go further into the understanding of the effect of roughness, it is necessary to separate the effect on the volume magnetostatic contribution from the effect on other volume

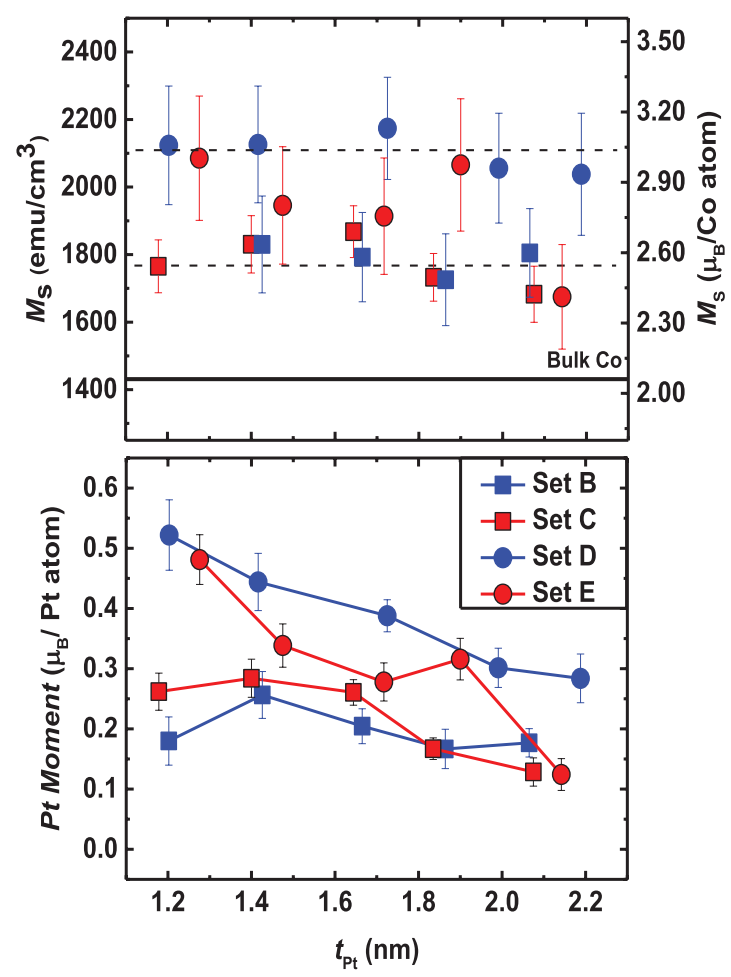

Figure 6. Top: saturation magnetization $M_{\mathrm{S}}$ versus the Pt thickness in $\left(\mathrm{Co}_{0.58 \mathrm{~nm}} / \mathrm{Pt}\left(t_{\mathrm{Pt}}\right)\right)$ multilayers from various sets $\mathrm{B}-\mathrm{E}$. The black horizontal line corresponds to bulk Co $\left(1430 \mathrm{emu} \mathrm{cm}^{-3}\right)$. The dashed lines are guides for eyes. Bottom: average induced $\mathrm{Pt}$ moment versus the Pt thickness in $\left(\mathrm{Co}_{0.58 \mathrm{~nm}} / \mathrm{Pt}\left(t_{\mathrm{Pt}}\right)\right)$ multilayers from various sets $\mathrm{B}-\mathrm{E}$.

and surface contributions to the anisotropy. All these contribute to the effective anisotropy discussed above. For this purpose, the saturation magnetization $\left(M_{\mathrm{s}}\right)$ has been carefully analyzed for all $(\mathrm{Co} / \mathrm{Pt})$ stackings. The $M_{\mathrm{s}}$ values per $\mathrm{Co} \mathrm{cm}^{3}$ extracted from the hysteresis loops are reported in figure 6 (top) as a function of Pt thickness for the various sets B-E, and compared to bulk Co $\left(1430 \mathrm{emu}^{-3}\right)$.

One can first notice that $M_{\mathrm{s}}$ does not exhibit any significant dependence versus the Pt thickness. The $K_{\text {eff }}$ variation observed for the samples belonging to a given set is thus not due to a change in the volume dipolar contribution but, as mentioned previously, is likely related to a change in the Néel surface term. For all sample sets, the magnetization is obviously larger than in bulk cobalt. This enhancement in magnetization $\Delta M$ appears to depend on the buffer layer: approximately $+24 \%$ for $(\mathrm{Co} / \mathrm{Pt})$ stackings sputtered on $\mathrm{Pt}$ (sets $\mathrm{B}$ and $\mathrm{C}$ ) and $+48 \%$ for $(\mathrm{Co} / \mathrm{Pt}$ ) stackings sputtered on $\mathrm{MgO}$ (set D). The values obtained for samples grown on $\mathrm{AlO}_{x}$ (set E) exhibit an intermediate scattered behavior that might be related to the poorer quality of $\mathrm{x}$-ray reflectivity fits and thus to a larger uncertainty in the Co thickness determination. The large magnetization enhancement in samples grown on oxide layers naturally gives rise to a large enhancement of the volume dipolar anisotropy term $\left(-2 \pi M_{\mathrm{s}}^{2}\right)$ that contributes to reduce the effective anisotropy and the PMA in sets D and E. In order to explore the effect of interface morphology on the anisotropy by excluding the effect on this volume dipolar 
part, the $K_{\text {eff }}$ values in sets D and E are calculated from measured data by assuming a saturation magnetization equal to those in sets B and C (approximately $300 \mathrm{emu} \mathrm{cm}^{-3}$ smaller than the measured magnetization). This analysis results in the values given by the star symbols in figure 5. Excluding the difference due to volume dipolar contributions in sets grown on Pt or on oxides thus leads to $K_{\text {eff }}$ values larger for samples grown on oxide. This suggests the occurrence of a supplemental positive contribution that would promote PMA and overcome the reduction of the Néel surface contribution. According to Bruno's model [20], the roughness can give rise to an effective positive dipolar surface anisotropy promoting PMA whose order of magnitude depends on the parameters characterizing the roughness (average deviation from the reference plane and correlation length) and of course on the magnetization value. The author points out that this dipolar surface anisotropy may not be negligible and might even become the main contribution to the anisotropy when the roughness is very important. In the current case, the large roughness and magnetization values measured for the samples grown on oxides most likely yield a large positive dipolar surface anisotropy. This contribution counterbalances the reduction of the Néel surface anisotropy and eventually results in anisotropy constants exceeding those obtained for $(\mathrm{Co} / \mathrm{Pt})$ grown on $\mathrm{Pt}$. One can notice that the anisotropy for the set grown on $\mathrm{AlO}_{x}$ (set E) remains smaller than for the set grown on $\mathrm{MgO}$ (set D), meaning that the reduction of the Néel surface anisotropy should be larger and/or that the dipolar surface anisotropy should be smaller for those samples. More information on the exact nature of the disorder occurring at the interfaces for those different sets would be required to go further into this analysis.

Concerning the origin of the observed magnetization enhancement compared to bulk Co, two main contributions can be suggested: (i) the enhancement of the Co magnetic moment itself and (ii) the occurrence of an induced magnetic moment on Pt atoms. Both phenomena are likely to occur as reported in previous studies on Co-based and Pt-based systems, specifically at the $\mathrm{Co} / \mathrm{Pt}$ interfaces [26, 40, 41]. Element-selective and depth-resolved analysis would thus be ideally required to draw a precise conclusion on the origin of this magnetization enhancement. The amplitude of $\Delta M$ and its dependence on the Pt thickness and buffer layer can be, however, interestingly discussed in comparison with previous results and in connection with the structural analysis. Most investigations of interfacial Co moments have been carried out by $\mathrm{x}$-ray magnetic circular dichroism and reveal an enhancement of the orbital moment $\Delta m_{\text {orb }} \approx+0.07 \mu_{\mathrm{B}} / \mathrm{Co}$ atom, essentially located in a single atomic interface layer and closely related to the Co $3 d-\mathrm{Pt} 5 d$ hybridization [15, 41]. In sputtered systems, those enhancements are determined to be $\Delta m_{\text {spin-eff }}=+0.1 \mu_{\mathrm{B}} /$ at. and $\Delta m_{\text {orb }}=+0.07 \mu_{\mathrm{B}} /$ at. In our case, the measured enhanced magnetization would correspond to approximately $+0.7 \mu_{\mathrm{B}} / \mathrm{Co}$ at. for sets $\mathrm{D}$ and $\mathrm{E}$ and $+0.35 \mu_{\mathrm{B}} / \mathrm{Co}$ at. for sets $\mathrm{B}$ and $\mathrm{C}$, i.e. much too large to be solely attributed to the increased Co moment. The occurrence of a Pt moment parallel to the Co one, induced by the hybridization between the Co3d and the Pt $5 d$ orbitals and by spin-orbit coupling in $\mathrm{Pt}$, is thus strongly suggested, as previously reported by several authors in $(\mathrm{Co} / \mathrm{Pt})$ multilayers [16, 26, 28, 29, 42, 43]. An average Pt moment decreasing from 0.15 to $0.03 \mu_{\mathrm{B}} / \mathrm{Pt}$ at. when increasing the Pt thickness has been measured in $(\mathrm{Co}(0.4 \mathrm{~nm}) / \mathrm{Pt}(\times \mathrm{nm}))(x \in(0.9-4) \mathrm{nm})$ [42] via spin absorption measurements at the $\mathrm{Pt} \mathrm{L}_{3}$ edge. The use of a depth-resolved technique (resonant $\mathrm{x}$-ray reflectometry) for a single $\mathrm{Co} / \mathrm{Pt}$ interface (roughness of $0.7 \mathrm{~nm}$ ) revealed an induced $\mathrm{Pt} 5 \mathrm{~d}$ magnetic polarization of $0.21 \mu_{\mathrm{B}} / \mathrm{Pt}$ at. at the interface, followed by an exponential decay within $1 \mathrm{~nm}$ [43]. Moreover, Knepper and Yang [28] recently suggested that Pt atoms in thin Pt layers sandwiched between two Co layers can be polarized by Co atoms located on both sides of Pt. In CoPt alloys, various studies have determined an induced Pt moment close to $0.35 \mu_{\mathrm{B}} / \mathrm{Pt}$ at [40].

In the present case, if one considers that the excess in magnetization $\Delta M$ only originates from the induced Pt moment, the average induced Pt magnetization $M_{\mathrm{Pt}}$, the total effective thickness where Pt atoms are polarized $t_{\mathrm{Pol}}$ and the number of cobalt layers $n(n=4)$ are related by the following expression:

$$
M_{\mathrm{Pt}} \times t_{\mathrm{Pol}}=\Delta M \times n t_{\mathrm{Co}}
$$

Given the $1 \mathrm{~nm}$ value reported for room temperature $\mathrm{Pt}$ polarized thickness at a Co/Pt interface [43], the Pt layers in our samples are presumably polarized over their entire thickness. The effective polarized part would thus correspond to the three Pt layers surrounded by Co layers and to an approximate $1 \mathrm{~nm}$ thick layer in the first (last) bottom (top) Pt: $t_{\mathrm{Pol}}=3 t_{\mathrm{Pt}}+1$. This permits us to calculate a rough estimation of the average Pt induced moment for the various samples (figure 6 bottom). The values of induced moments are similar [28] or slightly larger than those generally reported for similar systems. In contrast to a single $\mathrm{Co} / \mathrm{Pt}$ interface or to relatively distant interfaces, the close $\mathrm{Co} / \mathrm{Pt}$ interfaces in present samples with thin $\mathrm{Pt}$ layers likely promote larger induced moments, since Co atoms on each side of the Pt layer could contribute to the Pt polarization [28]. The induced $\mathrm{Pt}$ moment might also be enhanced in the case of CoPt alloying at the interfaces, a hypothesis that cannot be ruled out, and that should be further analyzed via other complementary experimental techniques.

It appears in figure 6 (bottom) that both the Pt thickness and the nature of the buffer layer affect the average induced Pt moment. As already mentioned for $M_{\mathrm{s}}$, the Pt moment is larger for $(\mathrm{Co} / \mathrm{Pt})$ stackings sputtered on $\mathrm{MgO}$ and $\mathrm{AlO}_{x}$ (circle symbols, sets D and E) than for stackings sputtered on Pt (square symbols, sets B and C). As for the effective anisotropy constant, this difference likely originates from different interface structures when changing the buffer layer, as attested by the different Co roughnesses (figure 2). Increased roughness and/or inter-mixing for interfaces obtained on the oxide buffers increase the quantity of Pt atoms in direct contact with Co atoms and thus likely increase the average Pt induced moment, and consequently the magnetization of the $(\mathrm{Co} / \mathrm{Pt})$ multilayers.

The decrease in the average induced $\mathrm{Pt}$ moment when increasing the Pt thickness can be easily explained by: (i) the 
decay of the $\mathrm{Pt}$ moment when going further from the $\mathrm{Co} / \mathrm{Pt}$ interface and (ii) the increased distance between the consecutive $\mathrm{Co} / \mathrm{Pt}$ interfaces so that $\mathrm{Pt}$ atoms close to one interface are less polarized by the second further interface. The role of interface morphology can be invoked as well. As the induced Pt moment is larger for the sets grown on oxide layers, those exhibiting rougher interfaces, it increases for small Pt thickness when the roughness also increases. This enhancement (on oxide buffers and for small Pt thicknesses) is thus likely correlated with the changes in interface morphology; it is, however, not possible at this point to discriminate between the possible effects of roughness, intermixing and alloying. A more accurate evaluation of these various effects would require a depth-sensitive analysis of the induced Pt moment.

\section{Summary}

In conclusion, the link between the magnetic and structural properties in $(\mathrm{Co} / \mathrm{Pt})$ multilayers has been carefully and directly analyzed via the use of X-ray reflectivity, with specific attention paid to the influence of the Pt thickness and to the nature of the buffer layer (metallic/oxide). The comparison between the different sets of $(\mathrm{Co} / \mathrm{Pt})$ multilayers highlights the prominent role played by the layer roughness, especially the Co layers roughness in the present case. Rough interfaces largely contribute to reduce the effective anisotropy, i.e. the PMA. This is observed for a given buffer layer where decreasing the Pt thickness tends to increase the roughness and reduce the PMA. It is also observed for a given Pt thickness when the growth mode on an intermediate thin oxide layer leads to rougher interfaces. One can, however, underline that PMA can still be achieved in these systems deposited on oxides, an important point for the development of spintronic devices.

Simultaneously, rough interfaces result in a strong magnetization enhancement due to the polarization of $\mathrm{Pt}$ atoms in direct contact with $\mathrm{Co}$ and to close $\mathrm{Co} / \mathrm{Pt}$ interfaces. The Pt induced polarization is particularly large when the growth is achieved on an oxide layer and increases when decreasing the Pt thickness. The influence of roughness on the Pt magnetization, i.e. on the volume dipolar contribution, appears to be the predominant factor in the reduction of effective anisotropy observed in the sets grown on oxides compared to those grown on Pt.

Beyond the major role of volume dipolar contribution that reduces PMA, the results strongly suggest that the large roughness observed for samples grown on oxide layers gives rise to a supplemental positive contribution promoting PMA, consistent with the dipolar surface anisotropy term developed by Bruno. In cases where the magnetization is strongly enhanced, this term becomes large enough to overcome the reduction in Néel surface anisotropy and an anisotropy exceeding the one obtained for $(\mathrm{Co} / \mathrm{Pt})$ deposited on $\mathrm{Pt}$ is achieved. In the long debate on the role of roughness/intermixing on PMA and more generally on anisotropy, these results bring further evidence that a good knowledge of interface structure is essential and that, beyond the expected reduction of Néel surface anisotropy, multiple and possibly opposite effects of roughness/intermixing have to be taken into account. Among these, contributions specifically related to roughness, such as dipolar surface terms, may be interestingly taken advantage of to enhance the PMA in systems with non-ideally sharp interfaces.

\section{Acknowledgments}

The authors acknowledge financial support from FEDER, the Region Lorraine, the Grand Nancy. The authors thank S Robert for the technical support and fruitful discussions about the reflectometry fitting procedure.

\section{References}

[1] Mangin S, Henry Y, Ravelosona D, Katine J A and Fullerton E E 2009 Appl. Phys. Lett. 94012502

[2] Boulle O, Malinowski G and Kläui M 2011 Mater. Sci. Eng. R 72159

[3] Fukami S, Suzuki T, Nagahara K, Ohshima N and Ishiwata N 2010 J. Appl. Phys. 108113914

[4] Néel L 1954 J. Phys. Radiat. 15225

[5] Zhang B, Krishnan K M, Lee C H and Farrow R F C 1993 J. Appl. Phys. $\mathbf{7 3} 6198$

[6] Chappert C 1988 J. Appl. Phys. 645736

[7] Gehanno V, Marty A, Gilles B and Samson Y 1997 Phys. Rev. B 5512552

[8] Avisou A, Dufour C and Dumesnil K 2008 J. Appl. Phys. 103 07E135

[9] Mougin A, Dufour C, Dumesnil K and Mangin P 2000 Phys. Rev. B 629517

[10] Chappert C, Le Dang K, Beauvillain P, Hurdequint H and Renard D 1986 Phys. Rev. B 343192

[11] Pescia D, Zampieri G, Stampanomi M, Bona G L, Willis R F and Meier F 1987 Phys. Rev. Lett. 58933

[12] Carcia P F 1988 J. Appl. Phys. 635066

[13] Carcia P F, Li Z G and Zeper W B 1993 J. Magn. Magn. Mater. 121452

[14] Daalderop G H O, Kelly P J and Den Broeder F J A 1992 Phys. Rev. Lett. 68682

[15] Weller D, Wu Y, Stöhr J, Samant M G, Hermsmeier B D and Chappert C 1994 Phys. Rev. B 49888

[16] Lin C-J, Gorman G L, Lee C H, Farrow R F C, Marinero E E, Do H V, Notarys H and Chien C J 1991 J. Magn. Magn. Mater. 93194

[17] Kim J and Shin S C 1996 J. Appl. Phys. 803121

[18] Hashimoto S, Ochiai Y and Aso K 1989 J. Appl. Phys. 664909

[19] Bertero G A, Sinclair R, Park C H and Shen Z X 1995 J. Appl. Phys. 773953

[20] Bruno P 1988 J. Appl. Phys. 643153

[21] Bruno P 1988 J. Phys. F: Met. Phys. 181291

[22] Macrander A T, Liu C, Csencsits R, Cook R, Kirk M and Headrick R 2000 Physica B 283157

[23] Rozatian A S H, Marrows C H, Hase T P A and Tanner B K 2005 J. Phys.: Condens. Matter 173759

[24] Grenet L, Arm C, Warin P, Pouget S, Marty A, Notin L and Jamet M 2011 (arXiv:1110.2628v1)

[25] Shan R, Gao T R, Zhou S M, Wu X S, Fang Y K and Han B S 2006 J. Appl. Phys. 99063907

[26] Canedy C L, Li X W and Xiao G 2000 Phys. Rev. B $\mathbf{6 2} 508$ 
[27] Liu Z Y, Zhang F, Chen H L, Xu B, Yu D L, He J L and Tian Y J 2009 Phys. Rev. B 79024427

[28] Knepper J and Yang F Y 2005 Phys. Rev. B 71224403

[29] Emori S and Beach G S D 2011 J. Appl. Phys. 110033919

[30] Parratt L G 1954 Phys. Rev. 95359

[31] Johnson M T, Bloemen P J H, den Broeder F J A and de Vries J J 1996 Rep. Prog. Phys. 591409

[32] Dumesnil K and Andrieu S 2013 Molecular Beam Epitaxy: From Research to Mass Production (Amsterdam: Elsevier) pp 487-507

[33] Tasker P W and Duffy D M 1984 Surf. Sci. 13791

[34] Kisielewski M, Maziewski A, Tekielak M, Ferré J, Lemerle S, Mathet V and Chappert C 2003 J. Magn. Magn. Mater. 260231

[35] Stillrich H, Menk C, Frömter R and Oepen H P 2010 J. Magn. Magn. Mater. 3221353
[36] Ter Lim S, Tran M, Chenchen J W, Ying J F, Han G, Ter Lim S, Tran M, Chenchen J W, Ying J F and Han G 2015 J. Appl. Phys. 117 17A731

[37] Lee T Y, Son D S, Lim S H and Lee S R 2013 J. Appl. Phys. 1132013

[38] Bertero G A and Sinclair R 1994 J. Magn. Magn. Mater. 134173

[39] Kim G 2008 Appl. Phys. Lett. 92172502

[40] Schütz G, Wienke R, Wilhelm W, Zeper W B and Ebert H 1990 J. Appl. Phys. 674456

[41] Nakajima N, Koide T, Shidara T, Miyauchi H, Fukutani H, Fujimori A, Katayama T, Nyvlt M and Suzuki Y 1998 Phys. Rev. Lett. 815229

[42] Rüegg S, Schütz G, Fischer P, Wienke R and Zeper W B 1991 J. Appl. Phys. 695655

[43] Geissler J, Goering E, Justen M, Weigand F, Schütz G, Langer J, Schmitz D, Maletta H and Mattheis R 2001 Phys. Rev. B 65020405 